\title{
O Psicólogo Apoiador Matricial: Percepções e Práticas na Atenção Básica
}


Resumo: Buscando nortear novas práticas em saúde, surge a proposta do apoio matricial como um arranjo organizacional cujo objetivo é fazer que uma equipe multiprofissional leve suporte assistencial e técnicopedagógico às equipes que compõem a Estratégia Saúde da Família. Observa-se a crescente inserção do psicólogo nesses serviços em virtude das dificuldades apresentadas pelos profissionais da Saúde da Família no lidar com as questões que envolvem a saúde mental dos usuários. Este estudo, de caráter descritivo, teve por objetivo verificar a percepção que os psicólogos apoiadores matriciais possuem sobre suas atividades e se estas correspondem às diretrizes estabelecidas pelo SUS. Foram entrevistados 10 psicólogos, com idade entre 23 e 45 anos. Os resultados mostraram que, apesar de haver tendência para adoção de novas práticas, os psicólogos ainda apresentam crenças e práticas enraizadas no modelo biomédico. O apoio matricial como dispositivo de mudança ainda não está bem compreendido por esses profissionais, o que demonstra que ainda há um longo caminho a percorrer nesse sentido.

Palavras-chave: Psicólogos. Apoio matricial. Saúde pública. Atuação do Psicólogo. Saúde da Família.

\begin{abstract}
Matrix support comprises an organizational arrangement that aims to bring assistance and technicalpedagogical support to the Family Health Strategy teams by a multi-professional team. This strategy aims to guide new practices in health. The growth of the number of psychologists in these services is observed, what is due to difficulties in dealing with the users' mental health problems according to family health practitioners. This descriptive study aims to investigate the matrix support psychologists' perceptions of their activities, and whether they correspond to the guidelines established by SUS. About 10 psychologists, with ages that range from 23 to 45 years old, were interviewed. The results demonstrate that despite there is a tendency for psychologists to adopt the new practices, they show beliefs and practices based on the biomedical paradigm. The matrix support as a device for change has not yet been applied by these professionals, revealing that there is still a long way to go in order to reach this ideal.
\end{abstract}

Keywords: Psychologists. Matrix support. Public health. Psychologist performance. Family health.

Resumen: Buscando nortear nuevas prácticas en salud, surge la propuesta del apoyo matricial como un arreglo organizacional cuyo objetivo es hacer que un equipo multiprofesional lleve soporte asistencial y técnico pedagógico a los equipos que componen la Estrategia Salud de la Familia. Se observa la creciente inserción del psicólogo en esos servicios en virtud de las dificultades presentadas por los profesionales de la Salud de la Familia en el lidiar con las cuestiones que involucran la salud mental de los usuarios. Este estudio, de carácter descriptivo, tuvo como objetivo verificar la percepción que los psicólogos apoyadores matriciales poseen sobre sus actividades y si éstas corresponden a las directrices establecidas por el SUS. Fueron entrevistados 10 psicólogos, con edad entre 23 y 45 años. Los resultados mostraron que, a pesar de haber inclinación para la adopción de nuevas prácticas, los psicólogos aún presentan creencias y prácticas arraigadas en el modelo biomédico. El apoyo matricial como dispositivo de cambio aún no está bien comprendido por esos profesionales, lo que demuestra que aún hay un largo camino a recorrer en ese sentido. Palabras clave: Psicólogos. Apoyo matricial. Salud pública. Actuación del Psicologo. Salud de la família.

Ao enfatizar a importância dos cuidados primários para o desenvolvimento de políticas públicas mais consistentes e eficazes, o Sistema Único de Saúde (SUS) parte da visão de que a saúde é muito mais que ausência de doença, pois envolve uma série de fatores relacionados às condições e aos modos de vida dos sujeitos, além de aspectos psicofisiológicos e socioculturais (Brasil, 2009). Essa visão ampliada do processo saúde-doença aponta sua complexidade Agradecimentos: $\mathrm{CNPq}$ Secretaria Municipal de Saúde de João Pessoa/PB mais para a doença (Tanaka \& Ribeiro, 2009).

A Atenção Primária ou, mais especificamente, a Atenção Básica - designação usada no Brasil (Fermino, Patrício, Krawulski, \& Sisson, 2009) - constitui a porta de entrada dos usuários ao SUS, e tem por objetivo reorganizar os serviços tendo como um dos seus preceitos o trabalho interdisciplinar e intersetorial, assumindo o cuidado com os usuários em caráter de integralidade (Brasil, 2006). Atualmente, os principais serviços e atividades nesse nível de atenção são conduzidos pela Estratégia Saúde da Família (ESF), composta por no mínimo um médico generalista, por 
um enfermeiro e por agentes comunitários de saúde. Entretanto, as diversas demandas existentes no dia a dia desses serviços têm mostrado a necessidade da incorporação de outros profissionais (Brasil, 2009).

Nesse sentido, o Ministério da Saúde, por meio da Portaria GM no 154, de 24 de janeiro de 2008 - republicada em 04 de março de 2008 - criou o Núcleo de Apoio à Saúde da Família (NASF), que tem por objetivo ampliar a abrangência e o foco das ações da Atenção Básica trabalhando em parceria com as equipes da ESF. Diferentemente destas, no entanto, o NASF não constitui porta de entrada. Sua função é apoiar o trabalho já desenvolvido pela ESF por meio de "atendimento compartilhado, com troca de saberes, capacitação e responsabilidades mútuas, gerando experiência para todos os profissionais envolvidos, mediante amplas metodologias, tais como estudo e discussão de casos e situações, projetos terapêuticos, orientações e atendimento conjunto, etc" (Brasil, 2009, p. 07). A interdisciplinaridade e a intersetorialidade são, assim, ferramentas essenciais para o desenvolvimento das atividades desses núcleos. Todavia, trabalhar com esse olhar ainda encontra inúmeros desafios nesse contexto, especialmente em virtude da herança do modelo biomédico, cujo saber-fazer fragmentado e verticalizante ainda rege a maioria das práticas em saúde no Brasil (Neves \& Aciole, 2011; Sala, Luppi, Simões, \& Marsiglia, 2011; Tófoli \& Fortes, 2007).

$\mathrm{Na}$ tentativa de superar tais deficiências, vários modelos e estratégias de ações têm sido desenvolvidos pelos estudiosos da saúde coletiva com o intuito de nortear novas práticas em saúde, ganhando destaque, nos últimos tempos, a proposta do apoio matricial (Campos, 1999; Cunha \& Campos, 2011; Tófoli \& Fortes, 2007). Esta surge como um novo arranjo organizacional, cujo objetivo é fazer que uma equipe de profissionais de diferentes áreas de conhecimento leve suporte assistencial e técnico-pedagógico às chamadas equipes de referência, ou seja, às equipes que compõem a ESF (Brasil, 2009; Campos \& Domitti, 2007; Cunha \& Campos, 2011). A ideia é fomentar a discussão e a ação sobre as diversas demandas que se apresentam, no sentido de aumentar a resolutividade das ações da ESF e de reforçar o cuidado integral aos usuários. Em síntese, o apoio matricial tem a finalidade de promover o diálogo com as equipes de referência trabalhando sobre situações/temáticas diversas, as quais, muitas vezes, passam despercebidas aos olhos dos profissionais das equipes de Saúde da Família ou não são bem compreendidas e/ ou trabalhadas por estes. A saúde mental, por exemplo, é uma delas (Brasil, 2009).

Seguindo as diretrizes da reforma psiquiátrica, a Política Nacional de Saúde Mental (PNSM) vem promover a substituição do cuidado das pessoas que sofrem com transtornos mentais do contexto excludente dos hospitais psiquiátricos por uma rede de serviços locais ou regionais, mais próximos da família e da comunidade. Tendo como principal instrumento os Centros de Atenção Psicossocial (CAPS), essa nova rede de cuidado objetiva construir espaços de inclusão e de cidadania, especialmente para aquelas pessoas com transtornos mais severos e persistentes e com longa história de internação (Brasil, 2005). Entretanto, para autores como Fermino et al. (2009), essa desinstitucionalização acaba exigindo maior compromisso e participação da família e da sociedade, o que pressupõe mudanças ideológicas, subjetivas e atitudinais. No que tange aos cuidados primários em saúde, por seu caráter de porta de entrada, exige maiores conhecimentos e intervenções mais consistentes e eficazes por parte de seus profissionais.

Considerando dados de prevalência internacionais (Brasil, 2009), no Brasil, cerca 
de $3 \%$ da população apresenta transtornos mentais severos e persistentes, enquanto $9 \mathrm{a}$ $12 \%$ apresentam transtornos mentais leves, que necessitam de cuidados eventuais. No contexto da Atenção Básica, "estudos desde a década de 80 revelam que entre $33 \%$ e $56 \%$ dos pacientes atendidos nesse nível assistencial podem apresentar sintomas de transtornos mentais comuns" (Brasil, 2009, p. 38). Todavia, pesquisas recentes têm mostrado a dificuldade dos profissionais da ESF de lidar com essas questões (Tanaka \& Ribeiro, 2009; Silva, Furegato, Costa Junior, 2003; Silveira \& Vieira, 2009).

Por essas razões, como profissional da saúde mental, é crescente a inserção do psicólogo nesse nível de atenção, seja na própria estratégia ou mesmo em equipes auxiliares como o NASF. Nesse último caso, seu trabalho sob a égide do apoio matricial se faz relevante, pois permite que seus conhecimentos e práticas atuem como suporte dos conhecimentos e da prática dos demais profissionais da Atenção Básica, promovendo-se o cuidado compartilhado aos usuários. Contudo, é sabido que a prática psicológica sempre foi caracterizada pela clínica tradicional, em que a maioria das ações realizadas costuma focar os usuários de maneira isolada em detrimento da influência que os aspectos histórico-culturais e socioeconômicos exercem sobre o processo saúde-doença desses indivíduos. Estudos realizados recentemente vêm apontar que, mesmo no contexto da Atenção Básica e da promoção à saúde, essas práticas tendem a ser mantidas (Carvalho, Bosi, \& Freire, 2009; Fermino et al., 2009; Freire \& Pichelli, 2010).

Diante, pois, das dificuldades que a ciência e a prática psicológica possuem com relação ao novo fazer em saúde, faz-se relevante conhecer como os psicólogos, inseridos no contexto dos cuidados primários, em especial, na estratégia do apoio matricial, percebem suas ações. Desse modo, este estudo teve como objetivo verificar a percepção que os psicólogos apoiadores matriciais possuem sobre suas atividades e se estas se mostram condizentes com os princípios e as diretrizes do NASF/SUS.

\section{Método}

Constituiu-se de um estudo descritivo de caráter exploratório, com ênfase na metodologia qualitativa. O campo de investigação foram os cinco distritos sanitários da cidade de João Pessoa-PB, sede das 10 equipes do NASF existentes no Município, cujo trabalho seguia as diretrizes do apoio matricial. Cada distrito continha duas equipes do NASF, e cada equipe continha um profissional de Psicologia. Participaram, assim, 10 psicólogos, com idade entre 23 e 45 anos. A coleta de dados se deu por meio de entrevista semiestruturada, gravada e depois transcrita, cujo objetivo foi levantar a percepção dos participantes acerca do seu trabalho como apoiador matricial. As análises das entrevistas foram feitas segundo a análise categorial temática proposta por Figueiredo (1993), em que o discurso dos participantes é considerado com base em categorias determinadas a partir dos temas suscitados nas entrevistas e processado em uma série de etapas, que envolvem desde a análise individual de cada entrevista até a análise das entrevistas como um todo. O estudo seguiu todos os preceitos éticos relativos à pesquisa com seres humanos, tendo sido certificado pelo Comitê de Ética em Pesquisa (CEP/CCS/ UFPB).

\section{Resultados e discussões}

A análise das entrevistas apresentou, segundo a proposta utilizada, uma classe temática referente à prática dos psicólogos nos cuidados primários, com base em três categorias discursivas que abarcaram desde as atividades realizadas por esses profissionais até a importância que assumem nesse novo cenário. 


\section{Categoria 01 - Atividades realizadas}

Nessa primeira categoria, atividades voltadas para a coletividade e realizadas fora dos consultórios foram apontadas pelos psicólogos como novos modos de atuação. Entre as principais, destacaram-se: a) atividades de cunho assistencial-formativo com vistas a fortalecer o desempenho das equipes de referência e o vínculo com os usuários, como oficinas, palestras e rodas de conversa, entre outras, b) atividades de caráter técnicoburocrático, ligadas à gestão das unidades de saúde, c) atividades intersetoriais, no sentido de fortalecer a rede de apoio, d) escuta psicológica, em casos necessários, e e) visitas domiciliares aos usuários:

"É um trabalho técnico-formativo... é parte técnica, burocrática, administrativa que a gente realiza e tem, também, uma função formativa, que abrange não só os usuários, mas as equipes... a gente leva vídeos, faz relatos de práticas... textos que a gente leva... a gente problematiza, conversa sobre os processos de trabalho, sociedade, processos de dominação, sobre o SUS, PSF" (part. 04).

"Faço algumas escutas... faço visitas em casos de saúde, quando as pessoas têm transtorno mental... a gente faz uma roda de conversa... a gente vai conversar sobre a rede de saúde mental do Município, porque as pessoas não conhecem... a gente faz trabalhos de formação, de discussão, com os trabalhadores... e também com os usuários... tentando discutir sobre concepções de família, de Estado, de sociedade, de organização comunitária... nós também somos educadores" (part. 15).

Como pode ser observado, as atividades realizadas por esses profissionais procuraram ir ao encontro do estabelecido pelo Ministério da Saúde sobre as diretrizes do NASF, respeitando, principalmente, os aspectos da ação interdisciplinar e da educação permanente em saúde (Brasil, 2009). Sob a égide do apoio matricial, os psicólogos entrevistados procuraram levar o suporte técnico-pedagógico para as equipes de referência e os usuários (Campos \& Domitti, 2007), mostrando ser essa estratégia fundamental para a ampliação do cuidado. Ao relatarem suas atividades dentro desse dispositivo, os psicólogos também mostraram a possibilidade de novos horizontes do fazer psicológico na Atenção Básica, de maneira que suas ações não estão presas, como se pensa, ao fazer tradicional, da clínica. Como colocou Campos, "nada contra o divã, mas temos certeza de que a clínica que almejamos para o serviço público não será somente construída em volta dele" (2001, p.101). Contudo, realizar tais atividades ou mesmo compreender esse novo jeito de atuar não foi uma tarefa simples para esses psicólogos. Ao falar de suas atividades, os psicólogos não puderam deixar de relatar as dificuldades em realizá-las e de entender o que seria essa proposta do apoio matricial.

\section{Categoria $02-\mathrm{O}$ apoio matricial}

A ideia de Apoio Matricial emergiu para esses profissionais como uma espécie de ponte, elo, interlocução, entre as relações estabelecidas pelos profissionais da ESF e os usuários. Como eles mesmos expuseram:

\footnotetext{
"A gente faz meio que uma coisa como uma ponte... a gente tá aqui pra apoiar... meio que pra ser um interlocutor, uma ponte para os serviços e a comunidade" (part. 02).

"O apoio é uma espécie de facilitador que ele tenta potencializar os trabalhos que já existem... tenta, por exemplo, criar outras práticas integrativas complementares, no sentido de se tornar mais viável o trabalho da Atenção Básica, principalmente pela Estratégia Saúde da Família" (part. 12).
}

Entretanto, esse novo fazer em saúde trouxe algumas inquietações para esse grupo de psicólogos, pois, ao exigir outro olhar da Psicologia sobre as questões de saúde, contrariou suas expectativas de atuação pautadas nos ensinamentos clínicocurativistas aprendidos em sua graduação: 
"Eu vinha da Academia com aquela visão de que seria clínica, que eu estaria num consultório atendendo, de maneira individual... no início, foi realmente difícil... a gente vem e se depara com uma realidade totalmente diferente, você não está preparada para aquilo... no início, a gente tem aquele choque" (part. 08). "Eu não sabia como ia ser o NASF, porque o NASF era uma coisa que ninguém sabia, e acho que na verdade ninguém sabe ainda direito o que é... eu achava que a gente ia poder ficar junto com todo mundo, com todos os profissionais para poder fazer todo o apoio clínico que precisasse" (part. 17).

A formação em Psicologia, ao privilegiar a clínica tradicional, trouxe algumas implicações para o trabalho do psicólogo em novos contextos, principalmente quando este se vê diante de demandas coletivas, como é o caso daquelas com que eles têm se deparado nos serviços públicos de saúde. Sabendo-se que a clínica psicológica tradicional não dá conta de tais demandas, nem é o tipo de intervenção que se espera nesse nível de atenção, os psicólogos entrevistados viram-se confusos com relação ao que deles se esperava dentro desse novo arranjo organizacional, confusão essa que não se restringiu apenas às suas expectativas, mas também às dos demais profissionais da ESF:

"A equipe também tinha essa visão de que nós iríamos pra lá e que nós iríamos fazer a parte clínica. Eles diziam: 'oba, nós temos um psicólogo aqui e nós temos várias demandas da Psicologia, e vocês vão fazer terapia...', e isso foi um trabalho um pouco difícil da gente meio que desmistificar" (part. 02).

"Quando eu cheguei pra trabalhar como apoiador... a expectativa com relação ao NASF que tinha a equipe de Saúde da Família era que a gente ia fazer um trabalho ambulatorial, de atendimento direto, o psicólogo ia atender clínica... teve essa expectativa muito grande... a gente percebeu que eles ficaram um pouco frustrados quando eles perceberam que o que a gente vinha fazer não era o que eles imaginavam" (part. 04)
A grande demanda em saúde mental que se apresenta no cotidiano das equipes de Saúde da Família (Brasil, 2009) e o seu despreparo em lidar com essas questões geram, segundo Bezerra e Dimenstein (2008), certo desconforto nos profissionais da ESF, o que os leva à prática de encaminhamentos excessivos. A inserção do psicólogo nesses serviços é vista assim, por esses profissionais, como uma solução para seus problemas. É justamente para trazer maior conhecimento e facilitar o trato desses profissionais com essas demandas, de forma a extinguir a demasiada busca pelas especialidades, que surge a proposta do apoio matricial (Brasil, 2009; Campos \& Domitti, 2007). Como visto, para que o psicólogo possa desenvolver suas práticas dentro dessa nova estratégia, não é só a sua visão que precisa ser reconstruída, mas também a visão dos profissionais da saúde com os quais ele trabalha. Para isso, reitera-se a necessidade da inserção cada vez maior dos psicólogos em equipes multi e interdisciplinares nesse contexto. Tal afirmativa remete à seguinte questão: qual a importância do psicólogo nessa nova prática de saúde?

\section{Categoria 03 - O papel do psicólogo apoiador matricial}

Ao apontarem sua diversidade de atividades, os psicólogos entrevistados também mostraram estar cientes de que esse novo fazer da Psicologia requer uma nova postura de seus profissionais. Desse modo, ressaltaram alguns aspectos acerca da importância de sua inserção nos serviços primários e do seu papel como apoiador matricial:

"Com relação ao meu trabalho enquanto psicólogo, alguém me falou uma vez que a Psicologia seria um instrumento de transformação... e dentro do SUS, a Psicologia pode ser esse instrumento de transformação, de problematização, de questionamento" (part. 04). 
Como já discutido, o cuidado em saúde oferecido pelos serviços primários requer um engajamento diferente de seus profissionais, diferente do lidar somente com as patologias já instaladas. Nesse novo contexto, o papel a desempenhar não é mais o de curador, mas o de agente de mudanças, que, segundo Dimenstein, deve ser construído "a partir de um compromisso social perante o ideário do sistema de saúde e seus usuários" (2001, p. 57). Isso significa dizer que, para os profissionais da saúde, o fundamental agora é serem protagonistas de mudanças sociais, cujas ações devem estimular e preparar os usuários a buscarem, por si só, condições dignas de viver, condições essas que envolvem não só o não adoecer mas também o viver com autonomia e qualidade. Para tanto, modificações na concepção que esses sujeitos possuem sobre o homem e a sociedade, bem como o tipo de compromisso que assumem perante os usuários e a sociedade, se fazem necessárias (Bock, 2009). Todavia, para os psicólogos entrevistados, essas questões ainda se mostraram atreladas ao pensamento individualista e naturalizante, especialmente no tocante às características subjetivas inerentes à profissão:

"A gente tem essa facilidade de lidar com o outro, de trabalhar a relação, de perceber algumas coisas que outras profissões não percebem... a gente tem aquele olhar, que algumas pessoas dizem: mal chegou e já está me analisando? Tem muito essa coisa, porque a gente tem essa tendência (...)" (part. 08).

“O psicólogo ele tem essa ferramenta mais aprofundada... ele tem uma habilidade maior de lidar com o comportamento... o psicólogo é fundamental, é relevante a participação do profissional de Psicologia, porque ele tem essa ferramenta mais aguçada pra entender isso, não que ele seja o melhor, mas ele tem essa habilidade de lidar melhor com essas temáticas mais profundas, que requer um olhar mais profundo" (part.12).

A visão do psicólogo como um profissional da subjetividade, que sabe lidar com o sofrimento, com as angústias e com os anseios do outro, é uma característica atribuída à categoria que foi construída tanto pela Academia quanto pela clínica psicológica tradicional. Essa particularidade de sua formação levou, pois, à crença de que cabe somente ao psicólogo desenvolver atividades em que se necessita de um olhar mais profundo sobre os sujeitos. A importância desses profissionais nos serviços públicos de saúde estaria ligada, assim, para esses psicólogos, pela facilidade que apresentam em adentrar no íntimo dos usuários, de maneira que, ao trabalharem com seus conflitos internos, estariam contribuindo para melhorias na qualidade de vida das populações. Tal questão é reforçada por esses profissionais diante do descaso relacionado às demandas do sofrimento psíquico. Com ênfase no corpo doente, nos sinais e nos sintomas das patologias mentais, o foco das ações em saúde ainda é centrado no uso exagerado de medicamentos e demais tecnologias duras (Merhy \& Franco, 2003). Frente a isso, possibilitar um espaço de escuta para esse sofrimento como forma de evitar o desenfreado sistema de medicamentação foi outro elemento tido como fundamental para esses psicólogos devido a sua importância nos serviços de Atenção Básica:

"A equipe cobrava muito... eles acham que é melhor ter um psicólogo pra fazer escuta qualificada... eles querem que tenha o psicólogo pra eles não fazerem essa escuta... tipo: ' bota o psicólogo pra fazer isso, que ele faz bem demais, eu é que não quero fazer' ... tem muito ainda dessa visão" (part. 08).

"Dentro da equipe básica, deveria ter a figura do psicólogo, do mesmo jeito que tem a figura do enfermeiro, do médico... aí poderia se ver que nem em todos os casos precisaria de medicamentos, bastando uma escuta qualificada, o que causaria uma redução na prescrição de medicamento, então tudo isso poderia melhorar" (part. 13).

No tocante a essa questão, é sabido que a escuta é uma técnica bastante utilizada nas 
práticas psicológicas e que, de certo modo, a formação em Psicologia delega uma grande responsabilidade e cuidado ao uso que dela se faz, principalmente quando estão envolvidas questões de ordem ética. No entanto, vale ressaltar que essa escuta, dentro da ciência psicológica, sempre foi moldada com base no cuidado individual, sendo delegado pouco interesse aos problemas históricos e sociais dos indivíduos. Nos serviços de saúde, essa escuta qualificada, que tanto se almeja e que é extensiva aos demais trabalhadores da saúde, vai muito além. Essa escuta ampliada, relacionada para alguns autores ao acolhimento (Takemoto \& Silva, 2007; Teixeira, 2003) ou ao próprio cuidado em saúde (Ayres, 2004), remete a um espaço dialógico que implica uma doação que não se restringe ao usuário, mas que envolve também o profissional que ouve. Isso significa dizer, de acordo com esses autores, que o ouvir, em saúde, traz algumas responsabilidades para seus trabalhadores, abarcando especialmente o que fazer com o que se ouviu. Desse modo, o ouvir necessário à construção dessas relações intersubjetivas (itálicos nossos) (Ayres, 2004) em saúde significa não só ouvir pela necessidade que o outro tem de falar, mas no sentido de conhecer, de dialogar com os usuários e de intervir segundo suas necessidades individuais e sociais. Essa forma de ouvir, no entanto, pouco ou quase nunca é trabalhada pelos aparelhos formadores, e, no caso dos cursos de Psicologia, não é diferente. Assim, é de forma errônea que esses psicólogos agregam sua importância ao uso dessa técnica, como forma de justificar a importância de seus serviços nos cuidados primários. Além disso, corroborando afirmações dadas por Bock, foi possível perceber algumas vezes, no discurso dos psicólogos, concepções grandiosas acerca do seu fazer e da sua profissão, o que acarreta sensações de onipotência frente às demais profissões:

\begin{abstract}
“O profissional de Psicologia é importante porque ele tem o poder dessa clínica ampliada, dessa escuta... que nos dá em determinados momentos, panos pras mangas... não que os outros profissionais não tenham, eles têm, só que de forma equivocada" (part. 12).

"O psicólogo, ele tem muito a questão da escuta... a gente pode estar ajudando nessa formação, nessa mudança mesmo de atitudes diante da vida, diante dos usuários, diante das pessoas, que os profissionais ainda não têm consolidado... a gente pode contribuir com esse processo das pessoas falarem, pra se colocarem no mundo... e a Psicologia é importante pra isso também, pra que as pessoas se sintam importantes, se sintam capazes mesmo de intervir" (part. 15).
\end{abstract}

Esse poder supremo de ajudar o outro, de trazer felicidade e equilíbrio para a vida das pessoas remete, para Bock, a algumas considerações sobre a forma como a Psicologia tem estruturado seu conhecimento ao longo dos anos e como esse saber tem influenciado suas práticas. Essa autora parte, por exemplo, da crença desses profissionais de que o mundo psicológico não é algo construído socialmente, mas que já "nasce conosco e tem destino traçado" (2009, p. 23). Diante dessa perspectiva naturalizante, o homem também é concebido e tratado apenas por meio de sua individualidade. A partir dessa visão, segundo essa autora, crê-se que de forma natural, cada indivíduo, por si só e de maneira singular, irá desenvolver-se, mas irá enfrentar, antes disso, uma série de obstáculos trazidos pelas suas condições de vida, como, por exemplo, doenças, conflitos, vulnerabilidades, etc. Para garantir que esse desenvolvimento ocorra sem maiores complicações, surge a figura do psicólogo, que assume, para tanto, o papel de facilitador desse processo. Assim, no campo da saúde, caberia aos próprios usuários do SUS a responsabilidade pela obtenção ou não de uma vida saudável, e, ao psicólogo, a função de mediador dessas escolhas:

"Eu não posso policiar um diabético ou um hipertenso, ver se ele tá comendo açúcar, 
mas eu tenho que levá-lo a entender que ele é o dono da vida dele e da saúde dele, então quem tem que se transformar é ele; a gente é apenas facilitador desse processo" (part. 12).

Essa missão sublime (itálicos nossos) (Bock, 2009, p. 24) atribuída à categoria e introjetada pela maior parte dos psicólogos faz que esses profissionais não enxerguem suas práticas como direcionadoras, e, por conseguinte, como alienadoras e/ou transformadoras, mas apenas como condutas neutras ao desenvolvimento natural dos seres humanos. Diante disso, esses dados também legitimam as conclusões dessa autora ao afirmar que a Psicologia construiu e ainda constrói sua prática somente por meio de intervenções técnicas, sem apontar, no entanto, "uma finalidade social e política para essa prática" (2009, p. 24). Isso demonstra que, por mais apta que a Psicologia pareça estar para atuar em serviços públicos, especialmente nos serviços de saúde, ainda se encontra distante de um verdadeiro compromisso social.

No que tange a esses aspectos, os psicólogos entrevistados, mesmo demonstrando uma visão enraizada e distorcida da sua importância nos cuidados primários, reconheceram a necessidade de rever sua prática, relacionando as possibilidades de transformação a partir de mudanças internas ao próprio sujeito profissional:

“O psicólogo tem que repensar sua prática... quando você problematiza e pensa do outro lado e vê as razões de outra metodologia, você repensa sua própria metodologia... é uma boa possibilidade para o psicólogo refletir sobre suas práticas, suas abordagens dentro da Psicologia comunitária e dentro desse campo maior que é a saúde pública e a Atenção Básica" (part. 04).

"Essa mudança tem que partir de nós, profissionais; por mais que o Ministério ou a Secretaria invista no profissional com capacitações, com cursos, se você não quer, não vai adiantar... apesar de todas as ferramentas que a gente tá tendo, todo incentivo, toda aposta que a gente tá tendo dessa gestão, se você não tiver uma mudança interna, não vai, entendeu?" (part. 13).

Observa-se, portanto, que a importância do psicólogo como apoiador matricial, mesmo tendo seu potencial transformador reconhecido pelos psicólogos entrevistados, ainda se apresentou, de maneira geral, atrelado à velha concepção do psicólogo clínico, cujas atividades tradicionais ainda se fazem importantes entre os demais conhecimentos.

\section{Considerações finais}

Visto que o saber-fazer psicológico tradicional não responde às demandas que se apresentam nos cuidados primários, transformações nas práticas dos psicólogos nesses serviços se fazem urgentes, caso se pretenda consolidar a Atenção Básica como novo campo profissional. A estratégia do apoio matricial se apresenta como um importante instrumento para essa consolidação, entretanto, muito ainda deve ser feito. No presente estudo, ao verificar a percepção de psicólogos apoiadores matriciais acerca de suas atividades, observou-se que, apesar de essas percepções mostrarem uma tendência convergente para o que foi estabelecido pelo Ministério da Saúde como diretrizes para essa estratégia, (Brasil, 2009), os psicólogos ainda apresentam algumas crenças e atitudes enraizadas no modelo clínico-biomédico. Como a formação em Psicologia ainda se mostra limitada e deficiente para as questões que envolvem as políticas sociais - neste caso, as políticas de saúde - os resultados apresentados mostraram que os psicólogos apoiadores matriciais enfrentaram dificuldades no desenvolvimento de novas atividades, especialmente porque estas não iam de encontro às suas expectativas e às dos demais profissionais com os quais eles trabalham, pautadas na atuação clínicoassistencialista. 
Desse modo, observa-se que o apoio matricial como dispositivo de mudança, ainda não está bem compreendido pelos profissionais da Atenção Básica. Longe de negar o seu valor - muito pelo contrário - percebe-se a necessidade de maiores esclarecimentos com relação a esse rearranjo organizacional no dia a dia dos serviços, de forma que apoiadores e equipe de referência consigam estruturar um verdadeiro cuidado aos usuários, com integralidade e responsabilidade mútua. Tais esclarecimentos também se justificam, no caso dos psicólogos em particular, pela necessidade de adotarem uma nova postura diante das questões de saúde, de maneira a poder atuar com compromisso social. Entretanto, é preciso refletir sobre qual tipo de compromisso social é necessário construir. Como visto neste estudo, a simples visão dos psicólogos de que a importância de sua inserção nos serviços públicos se deve à falta de espaços e à condição de poder ouvir o outro não envolve o compromisso da profissão com as políticas de saúde.

O compromisso social da Psicologia que se almeja construir, segundo autores como
Bock e Yamamoto (2007), não remete apenas à abrangência de sua atuação, ou seja, não significa apenas chegar às classes subalternas, mas significa ação, problematização, significa questionar suas condições de trabalho e intervenções de forma a produzir mudanças nas realidades dos usuários, o que remete a uma visão politizada de gerir autonomia. Para tanto, é preciso mais do que oferecer um serviço que se sabe necessário, como é o caso do apoio às equipes da ESF, é mais do que ajudar o outro a encontrar o equilíbrio e o bem-estar, mas reconhecer que sua atuação influencia atitudes e que a maneira como se percebe e se age no mundo repercute no olhar e no agir de outras pessoas, especialmente se estas estão sob seus cuidados. Estabelecer um verdadeiro compromisso social é colocado, pois, para os autores anteriormente citados, como um dos principais desafios da Psicologia nos serviços básicos, e o dia a dia tem mostrado que ainda há um longo caminho a percorrer nesse sentido. 


\section{Francisca Marina de Souza Freire}

Mestre e doutoranda em Psicologia Social pela Universidade Federal da Paraíba, João Pessoa - PB - Brasil.

E-mail: marinasfreire@hotmail.com

\section{Ana Alayde Werba Saldanha Pichelli}

Doutora em Psicologia pela Universidade de São Paulo e professora do programa de pós-graduação em Psicologia Social da Universidade Federal da Paraíba, João Pessoa - PB - Brasil.

E-mail: analayde@terra.com.br

Endereço para envio de correspondência:

Rua Desportista Manoel Gomes, 52, Jardim Cidade Universitária. CEP: 58051-660. João Pessoa, PB

Recebido 27/04/2012, 1a Reformulação 16/11//2012, Aprovado 17/12/2012. 


\section{Referências}

Ayres, J. R. C. de M. (2004). O cuidado, os modos de ser (do) humano e as práticas de saúde. Revista Saúde e Sociedade, 3(13), 16-29.

Bezerra, E., \& Dimenstein, M. (2008). Os CAPS e o trabalho em rede: tecendo o apoio matricial na atenção básica. Psicologia: Ciência e Profissão, 28(3), 632-645.

Bock. A. M. B. (2009). Psicologia e sua ideologia: 40 anos de compromisso com as elites. In A. M. B. Bock (Org.), Psicologia e o compromisso social (2a. ed., pp. 15-28). São Paulo: Cortez.

Brasil. Ministério da Saúde. Secretaria de Atenção à Saúde. Coordenação Geral de Saúde Mental. (2005). Reforma psiquiátrica e política de saúde mental no Brasil. Documento apresentado à Conferência Regional de Reforma dos Serviços de Saúde Mental: 15 anos depois de Caracas. OPAS. Brasília. Recuperado em 10 fev., 2012, de http://bvsms.saude.gov.br/ bvs/publicacoes/Relatorio15_anos_Caracas.pdf

Brasil. Ministério da Saúde. Secretaria de Atenção à Saúde. Departamento de Atenção Básica. (2006). Política Nacional de Atenção Básica. 60 p. (Série A. Normas e Manuais Técnicos, Série Pactos pela Saúde, v. 4). Brasília, DF.

Brasil. Ministério da Saúde. Portaria no 154, de 24 de janeiro de 2008 (2008). Cria os Núcleos de Apoio à Saúde da Família NASF. Recuperado em 08 fev., 2012, de http://pt.scribd.com/ doc/3665958/PORTARIA-15408-NASF

Brasil. Ministério da Saúde. Secretaria de Atenção à Saúde. Departamento de Atenção Básica. (2009). Diretrizes do NASF. 160 p. (Série B. Textos Básicos de Saúde, Cadernos de Atenção Básica, n. 27). Brasília, DF.

Campos, G. W. S., \& Domitti, A. C. (2007). Apoio matricial e equipe de referência: uma metodologia para o trabalho interdisciplinar em saúde. Cadernos de Saúde Pública, 23(2), 399-407.

Campos, G. W. S. (1999). Equipes de referência e apoio especializado matricial: um ensaio sobre a reorganização do trabalho em saúde. Ciência \& Saúde Coletiva, 4(2), 393-303.

Campos, R. O. (2001). Clínica: a palavra negada - sobre as práticas clínicas nos serviços substitutivos de saúde mental. Saúde em Debate, 25(58,) 98-111.

Carvalho, L. B., Bosi, M. L. M., \& Freire, J. C. (2009). A prática do psicólogo em saúde coletiva: um estudo no município de Fortaleza (CE), Brasil. Psicologia: Ciência e Profissão, 29(1), 60-73.

Cunha, G. T., \& Campos, G. W. de S. (2011). Apoio matricial e Atenção Primária em Saúde. Saúde e Sociedade, 20(4), 961-960.

Dimenstein, M. (2001). O psicólogo e o compromisso social no contexto da saúde coletiva. Psicologia em Estudo, 6(2), 57-63.

Fermino, J. M., Patrício, Z. M., Krawulski, E., \& Sisson, M. C. (2009). Atuação de psicólogos no Programa de Saúde da Família: o cotidiano de trabalho oportunizando repensar a formação e as práticas profissionais. Aletheia, 30, 113-128.
Freire, F. M. S., \& Pichelli, A. A. W. S. (2010). Princípios norteadores da prática psicológica na Atenção Básica: em busca da integralidade. Psicologia: Ciência e Profissão, 30(4), 840-853.

Figueiredo, M. A. C. (1993). Profissionais de saúde e Aids. Um estudo diferencial. Medicina, 26(3), 393-407.

Merhy, E. E., \& Franco, T. B. (2003). Por uma composição técnica do trabalho centrada no campo relacional e nas tecnologias leves. Saúde em Debate, 27(65), 316-323.

Neves, L. M. T., \& Aciole, G. G. (2011). Desafios da integralidade: revisitando as concepções sobre o papel do fisioterapeuta na equipe de Saúde da Família. Interface: Comunicação, Saúde e Educação, 15(37), 551-564.

Sala, A., Luppi, C. G., Simões, O., \& Marsiglia, R. G. (2011). Integralidade e Atenção Primária à Saúde: avaliação na perspectiva dos usuários de unidades de saúde do município de São Paulo. Saúde e Sociedade, 20(4), 448-460.

Silva, M. C. F., Furegato, A. R. F., \& Costa Júnior, M. L. (2003). Depressão: pontos de vista e conhecimento de enfermeiros da rede básica de saúde. Revista Latino-americana de Enfermagem, 11(1), 7-13.

Silveira, D. P., \& Vieira, A. L. S. (2009). Saúde mental e atenção básica em saúde: análise de uma experiência no nível local. Ciência \& Saúde Coletiva, 14(1), 139-148.

Takemoto, M. L. S., \& Silva, E. M. (2007). Acolhimento e transformações no processo de trabalho de enfermagem em unidades básicas de saúde de Campinas, São Paulo, Brasil. Cadernos de Saúde Pública, 23(2), 331-340.

Tanaka, O. Y., \& Ribeiro, E. L. (2009). Ações de saúde mental na atenção básica: caminho para ampliação da integralidade da atenção. Ciência \& Saúde Coletiva, 14(2), 477-486.

Teixeira, R. R. (2003). O acolhimento num serviço de saúde entendido como uma rede de conversações. In R. Pinheiro \& R. A. Mattos (Org), Construção da integralidade: cotidiano, saberes e práticas em saúde (pp. 89-112). Rio de Janeiro: UERJ, IMS, ABRASCO.

Tófoli, L. F., \& Fortes, S. (2007). Apoio matricial de saúde mental na atenção primária no município de Sobral/CE: um relato da experiência. SANARE: Revista de Políticas Públicas de Sobral/ $C E, 6(2), 34-42$.

Yamamoto, O. H. (2007). "Políticas sociais, 'terceiro setor' e 'compromisso social': perspectivas e limites do trabalho do Psicólogo. Psicologia \& Sociedade, 19(1), 30-37. 